\title{
Et si tout n'était qu'une question de perception : incidence des stresseurs sur la santé des travailleurs
}

\author{
Normand Laplante, Ph. D., Éric Gosselin, Ph. D. et Martin Lauzier, M. Sc. \\ Université du Québec en Outaouais
}

\section{INTRODUCTION}

Les travailleurs se disent de plus en plus stressés, situation à laquelle ne sont pas étrangères les profondes transformations qui ont marqué le monde du travail ces quelques dernières années (p. ex. restructuration d'entreprises, changements technologiques et organisationnels, intensification du travail, etc.). Au fil des années, les préoccupations pour la santé mentale au travail sont ainsi devenues de plus en plus criantes par les conséquences qui se font sentir tant sur les travailleurs que sur les organisations et la société dans son ensemble. Si le problème du stress professionnel se pose de façon de plus en plus évidente, l'action n'en est pas pour autant simplifiée.

\section{Au fil des années, les préoccupations pour la santé mentale au travail sont ainsi devenues de plus en plus criantes par les conséquences qui se font sentir tant sur les travailleurs que sur les organisations et la société dans son ensemble.}

Les risques du travail plus traditionnels comme ceux posés par des niveaux élevés de bruit, les contaminants chimiques ou les contraintes thermiques, pour ne nommer que ceux-là, ont une nature objective et sont facilement mesurables de façon précise. La situation est plus complexe pour les risques psychosociaux et les stresseurs en milieu de travail dont le caractère plus subjectif est indéniable et qui peuvent engendrer une situation de stress pour l'individu et ainsi affecter son état de santé. Ceci ne signifie pas que l'action pour contrôler les effets des stresseurs organisationnels ne doit être orientée que sur les individus, bien au contraire, alors qu'une action directe sur l'environnement de travail demeure toujours sou- haitable. Il devient néanmoins important de mieux comprendre comment les risques psychosociaux peuvent affecter la santé des individus. C'est ce que nous nous proposons de faire dans cet article alors que nous évaluons l'effet médiateur du stress psychologique, c'est-à-dire son rôle d'intermédiaire entre des stresseurs organisationnels et des indicateurs de santé. En d'autres termes, nous évaluons la mesure avec laquelle les stresseurs organisationnels agissent sur la santé des individus, mais en haussant leur niveau de stress plutôt qu'en affectant directement leur état de santé. Ainsi, face à une situation donnée, l'individu qui vit un plus haut niveau de stress va en ressentir des effets plus marqués sur sa santé contrairement à celui dont le niveau de stress est moindre. C'est ce que nous mesurons en nous basant sur une étude effectuée dans un centre hospitalier du Québec dont des résultats plus détaillés ont été publiés dans les actes du congrès de l'Association internationale de psychologie du travail de langue française ${ }^{1}$.

Les risques du travail plus traditionnels comme ceux posés par des niveaux élevés de bruit, les contaminants chimiques ou les contraintes thermiques, pour ne nommer que ceux-là, ont une nature objective et sont facilement mesurables de façon précise. La situation est plus complexe pour les risques psychosociaux et les stresseurs en milieu de travail dont le caractère plus subjectif est indéniable et qui peuvent engendrer une situation de stress pour l'individu et ainsi affecter leur état de santé. 


\section{STRESSEURS ET STRESS AU TRAVAIL}

\section{Les enquêtes sur les problématiques reliées au stress au travail démontrent un accroissement des problèmes de santé mentale et d'absentéisme mettant ainsi une pression accrue sur les régimes d'assurance-salaire et ceux d'indemnisation pour les lésions professionnelles.}

La problématique du stress au travail n'est certes pas nouvelle, mais elle devient de plus en plus au cœur des préoccupations notamment par les effets qui se font sentir tant sur les individus que sur les organisations. Les enquêtes sur les problématiques reliées au stress au travail démontrent un accroissement des problèmes de santé mentale et d'absentéisme mettant ainsi une pression accrue sur les régimes d'assurancesalaire et ceux d'indemnisation pour les lésions professionnelles $^{2}$.

\subsection{Une définition du stress}

Avant d'aller plus loin, afin d'éviter la confusion, il est important de préciser ce que nous entendons par le terme de stress. En effet, dans le langage courant, le terme de stress a été utilisé de façon variable en faisant parfois référence aux conditions du milieu, parfois aux réactions des individus face à ces conditions et souvent aux conséquences sur leur santé. Il importe donc de mieux préciser ce que signifie le stress. Celui-ci correspond à la réaction de l'individu qui doit réagir à une exigence élevée ou une menace de son environnement.

L'individu ressentira du stress lorsqu'il perçoit que ses capacités ou ses ressources sont insuffisantes pour s'adapter aux exigences de son environnement. Le stress est ainsi vécu au moment où l'individu est confronté à ces exigences, même si ses effets peuvent perdurer dans le temps.

L'individu ressentira du stress lorsqu'il perçoit que ses capacités ou ses ressources sont insuffisantes pour s'adapter aux exigences de son environnement. Le stress est ainsi vécu au mo- ment où l'individu est confronté à ces exigences, même si ses effets peuvent perdurer dans le temps.

Les stresseurs environnementaux font référence aux exigences de l'environnement, alors que le stress est la réaction de l'individu face à ces mêmes exigences. Les effets du stress sur la santé peuvent se faire sentir autant sur le plan physiologique que psychologique ou comportemental. Par ailleurs, étant donné le caractère subjectif du stress, celui-ci ne peut être évalué en se fondant uniquement sur les caractéristiques des stresseurs.

Face à des situations identiques potentiellement stressantes, le stress ressenti variera ainsi selon les individus alors que certains se sentiront très stressés et d'autres beaucoup moins. Les effets des stresseurs organisationnels sur la santé des individus ne sont plus à démontrer puisqu'ils sont bien documentés et se feraient sentir tant sur la santé physique que mentale et ce, non seulement à court terme, mais également à long terme. De plus, les stresseurs chroniques de plus faible intensité qui se répètent de façon continue sont tout aussi nocifs, sinon plus que les stresseurs aigus de forte intensité qui se manifestent de façon ponctuelle.

De plus, les stresseurs chroniques de plus faible intensité qui se répètent de façon continue sont tout aussi nocifs, sinon plus que les stresseurs aigus de forte intensité qui se manifestent de façon ponctuelle.

\subsection{L'action des stresseurs organisationnels}

L'environnement de travail semble ainsi de plus en plus stressant ou en d'autres termes, les stresseurs organisationnels liés au milieu de travail sont de plus en plus présents. Bon nombre d'études se sont intéressées à définir plus précisément ce que sont ces stresseurs organisationnels, même si ceux-ci ne sont pas faciles à mesurer. L'on note l'existence de deux modèles théoriques pour établir les liens de causalité entre les stresseurs organisationnels et la santé, soit le modèle de Karasek ${ }^{3}$ qui combine 
les effets de la demande psychologique et de l'autonomie auquel est venu s'ajouter le support social et le modèle de Siegrist ${ }^{4}$ qui se fonde sur le déséquilibre entre les efforts investis dans le travail et la reconnaissance. Le modèle de Karasek postule qu'un faible support social amplifie les effets nocifs de l'interaction entre une demande psychologique élevée et une faible autonomie. Selon le modèle de Siegrist, le déséquilibre ressenti par les individus entre les efforts consentis dans le travail et la reconnaissance qu'ils en retirent engendrerait une tension psychologique produisant des effets nocifs sur leur santé.

\section{Selon le modèle de Siegrist, le déséquilibre ressenti par les individus entre les efforts consentis dans le travail et la reconnaissance qu'ils en retirent engendrerait une tension psychologique produisant des effets nocifs sur leur santé.}

Ces modèles permettent de mieux comprendre les associations entre les stresseurs organisationnels et la santé tant physique que mentale en s'intéressant plus spécifiquement à certaines composantes plus significatives de l'environnement psychosocial de travail. Certaines critiques ont été adressées relativement aux deux modèles considérant qu'ils s'intéressaient à un nombre trop limité de variables et n'étaient pas suffisamment spécifiques du contexte de travail étudié. Ainsi, certains auteurs ont voulu élargir le spectre des stresseurs organisationnels pour englober d'autres facteurs que ceux considérés dans les modèles de Karasek et Siegrist. Un constat se dégage de ces études, les stresseurs organisationnels sont généralement reliés à des conditions spécifiques de la tâche (p. ex. surcharge de travail), à l'ambiguïté et au conflit de rôle, aux conflits interpersonnels, à l'environnement de travail non sécuritaire pour ne nommer que ces dimensions.
Au-delà de la définition des stresseurs organisationnels, la question demeure sur leurs mécanismes d'action, c'est-à-dire comment ils agissent pour influencer l'état de santé. Plusieurs études qui se sont inspirées des modèles de Karasek ou de Siegrist privilégient une action directe des stresseurs sur l'état de santé des personnes exposées. Autrement dit, les stresseurs organisationnels affecteraient directement l'état de santé des personnes qui leur sont exposées. Une autre approche de l'action des stresseurs organisationnels sur la santé fondée sur les travaux de Lazarus $^{5}$ examine plutôt la relation entre l'individu et son environnement comme s'influençant mutuellement où l'individu est un agent actif qui peut interpréter la situation dans laquelle il se trouve et agir pour s'y adapter ou la modifier.

Le stress naîtrait lorsque l'individu ne peut s'adapter ou modifier son environnement, soit lorsqu'il considère que sa relation avec son environnement dépasse ses ressources et représente une menace à son bien-être. Selon cette perspective transactionnelle, la perception des individus serait fondamentale alors que les stresseurs organisationnels influenceraient la santé des individus non par une action directe, mais plutôt par l'intermédiaire du stress ressenti par ceux-ci lorsqu'ils doivent faire face à des situations potentiellement stressantes. Certains auteurs ont voulu mesurer les effets médiateurs $\mathrm{du}$ stress entre les stresseurs organisationnels et la santé des individus, avec toutefois des résultats variables ${ }^{6}$.

Le stress naîtrait lorsque l'individu ne peut s'adapter ou modifier son environnement, soit lorsqu'il considère que sa relation avec son environnement dépasse ses ressources et représente une menace à son bien-être.

\section{LES EFFETS MÉDIATEURS DU STRESS PSYCHOLOGIQUE}

C'est dans ce contexte que nous avons voulu analyser la relation entre des stresseurs organisationnels et la santé des travailleurs en tenant compte de l'effet médiateur du stress perçu. La figure 1 illustre le modèle d'analyse retenu dans cette étude. L'effet direct des stresseurs organisationnels est représenté par la relation $\mathrm{C}$. L'effet médiateur du stress psychologique est 
représenté successivement par la relation A (effet des stresseurs organisationnels sur le stress psychologique) et la relation B (effet du stress

psychologique sur les indicateurs de santé).

Figure 1 : Modèle conceptuel de l'effet médiateur du stress psychologique

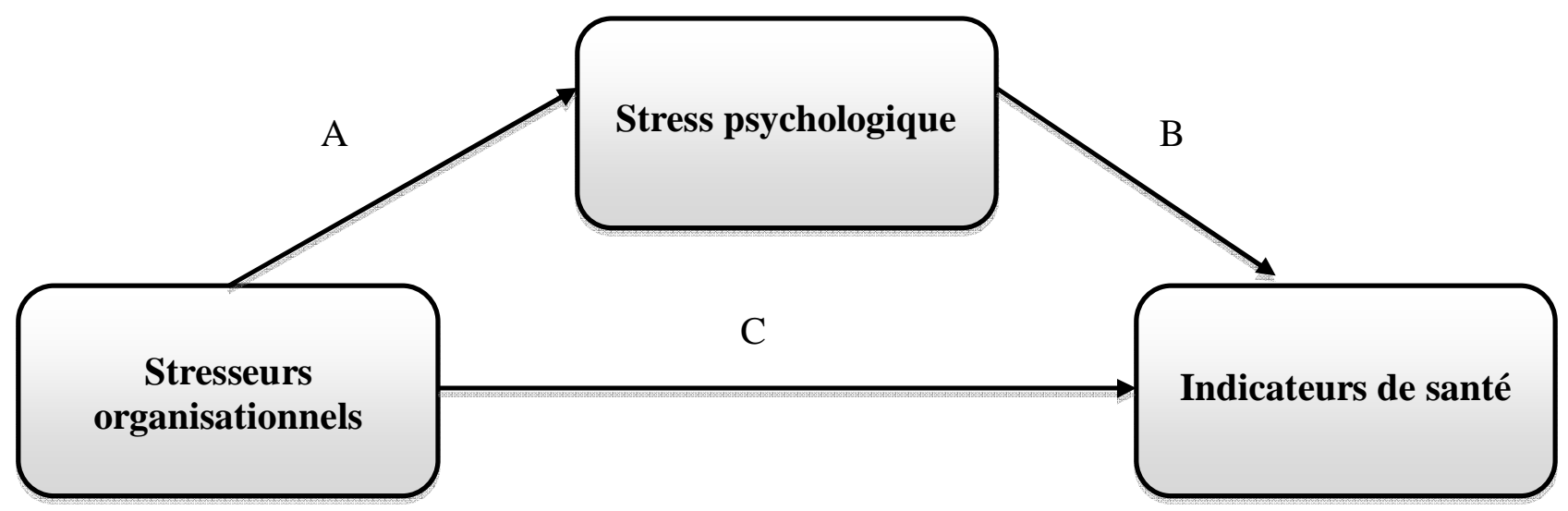

Le modèle conceptuel se base en premier lieu sur le fait que les stresseurs organisationnels influencent les indicateurs de santé (relation $\mathrm{C}$ significative). Tel que mentionné, de telles relations ont été observées dans plusieurs études. Pour évaluer un effet médiateur du stress psychologique, il faut également que les stresseurs organisationnels influencent le stress psychologique. C'est dire qu'il faut qu'en présence des stresseurs organisationnels, les individus ressentent un niveau de stress plus élevé (relation A significative). En contrepartie, il faut également que le stress psychologique influence les indicateurs de santé (relation B significative). Ainsi, les individus qui ressentent un plus haut niveau de stress verront leur état de santé plus affecté que ceux qui ressentent un niveau de stress plus faible. Finalement, lorsqu'on mesure la relation entre les stresseurs organisationnels et les indicateurs de santé, il faut que la relation $\mathrm{C}$ devienne plus faible (médiation partielle) ou s'annule (médiation totale) si l'on tient compte des effets du stress psychologique. En d'autres termes, les effets des stresseurs organisationnels passeraient en partie (médiation partielle) ou en totalité (médiation totale) par les relations A et B plutôt que par la relation $\mathrm{C}$ (effet direct).

\section{UNE RECHERCHE DANS LE SECTEUR DE LA SANTÉ}

\section{Le secteur de la santé est intéressant pour une telle étude car il est reconnu comme offrant des conditions de travail difficiles et potentiellement stressantes, notamment dans un contexte de pénurie de main- d'œuvre et des nombreuses restructurations qu'il a connues.}

Pour vérifier l'effet médiateur du stress psychologique, nous avons réalisé une étude au sein d'un établissement du secteur de la santé. Le secteur de la santé est intéressant pour une telle étude car il est reconnu comme offrant des conditions de travail difficiles et potentiellement stressantes, notamment dans un contexte de pénurie de main-d'œuvre et des nombreuses restructurations qu'il a connues. Un questionnaire a été distribué à l'ensemble du personnel de l'établissement à l'exception des médecins. Les catégories d'emploi visées par l'étude incluent ainsi le personnel infirmier, administratif, professionnel et technique. Un peu plus de $30 \%$ des personnes visées ont répondu au questionnaire pour un effectif valide de près de 700 personnes. Nous avons utilisé des échelles de mesure qui existaient déjà et dont la validité a été démontrée tant pour les stresseurs organisationnels que le stress psychologique et les indicateurs de santé. 


\subsection{La mesure des variables pertinentes pour la recherche}

Les questions sur les stresseurs organisationnels ont permis de recueillir les informations sur différentes caractéristiques du travail au sein de l'établissement. Aux fins de l'étude, nous avons réparti les stresseurs organisationnels en fonction de deux dimensions importantes du travail.

La première dimension porte sur des facteurs comme la charge de travail, les iniquités salariales, les demandes contradictoires, la rapidité d'exécution, le contexte d'urgence, le travail auprès des usagers, les risques de contamination ou de violence. Cette dimension du travail, appelée les stresseurs extrinsèques du travail, réfère aux exigences et au contexte dans lequel le travail se réalise. La seconde dimension, appelée les stresseurs intrinsèques du travail, porte plutôt sur le contenu et la mobilisation des capacités des individus dans la réalisation de ce travail. Elle réfère à des facteurs comme l'autonomie dans l'organisation du travail, l'utilisation de ses habiletés, les possibilités de planifier sa carrière et la participation à la prise de décisions.

Les stresseurs extrinsèques font ainsi référence à des conditions plus objectivées du travail selon les conditions qui sont imposées aux travailleurs. De leur côté, les stresseurs intrinsèques recèlent une dimension subjective plus importante alors que la difficulté d'utiliser ses habiletés ou de faire preuve d'autonomie et d'initiatives deviennent des stresseurs potentiels. Le partage des stresseurs organisationnels entre stresseurs extrinsèques et stresseurs intrinsèques a été validé par une analyse factorielle.

Le stress psychologique a été évalué à partir d'une échelle basée sur une série de questions relatives au vécu du travailleur au cours du dernier mois (échelle de stress perçu; PSS-14). À titre d'exemple, les questions vérifiaient dans quelle mesure la personne s'était sentie nerveuse et stressée, ou encore dans quelle mesure elle avait été capable de maîtriser son énervement. Deux indicateurs de santé ont été utilisés. Le premier est un indice de psychosomatisation basé sur la présence de symptômes physiques (p. ex. tremblement des mains, périodes d'étourdissement) au cours du dernier mois. Le deuxième indicateur est l'indice de détresse psychologique utilisé dans de nombreuses études dont les enquêtes sociale et de santé menées au Québec. Ces deux indicateurs de santé ne visent pas à établir un diagnostic de maladie, mais peuvent néanmoins constituer des signes précurseurs de problèmes de santé à plus long terme.

Les infirmières représentent une catégorie professionnelle ayant un fort pourcentage de personnes présentant une détresse psychologique élevée. Elles ne sont pas les seules à être touchées par le phénomène. D'autres catégories professionnelles comme les employés de bureau, le personnel des services techniques et le personnel cadre alors que dans ces catégories, plus de $40 \%$ des personnes présentent aussi une détresse psychologique élevée.

À titre d'exemple, il est reconnu que $20 \%$ de la population en général présente une détresse psychologique élevée. C'est toutefois le cas de $42,5 \%$ des travailleurs de notre étude, soit une proportion deux fois plus élevée que dans la population en général. Des pourcentages aussi élevés de détresse psychologique dans le secteur de la santé ont déjà été observés dans d'autres études confirmant l'importance du problème dans ce secteur d'activité.

Il est à noter que dans notre étude, les infirmières représentent une catégorie professionnelle ayant un fort pourcentage de personnes présentant une détresse psychologique élevée. Elles ne sont pas les seules à être touchées par le phénomène. D'autres catégories professionnelles comme les employés de bureau, le personnel des services techniques et le personnel cadre alors que dans ces catégories, plus de $40 \%$ des personnes présentent aussi une détresse psychologique élevée. En bref, le modèle de cette recherche est opérationnalisé dans un cadre d'analyse illustré à la figure 2 . 
Figure 2 : Opérationnalisation du modèle de l'effet médiateur du stress psychologique

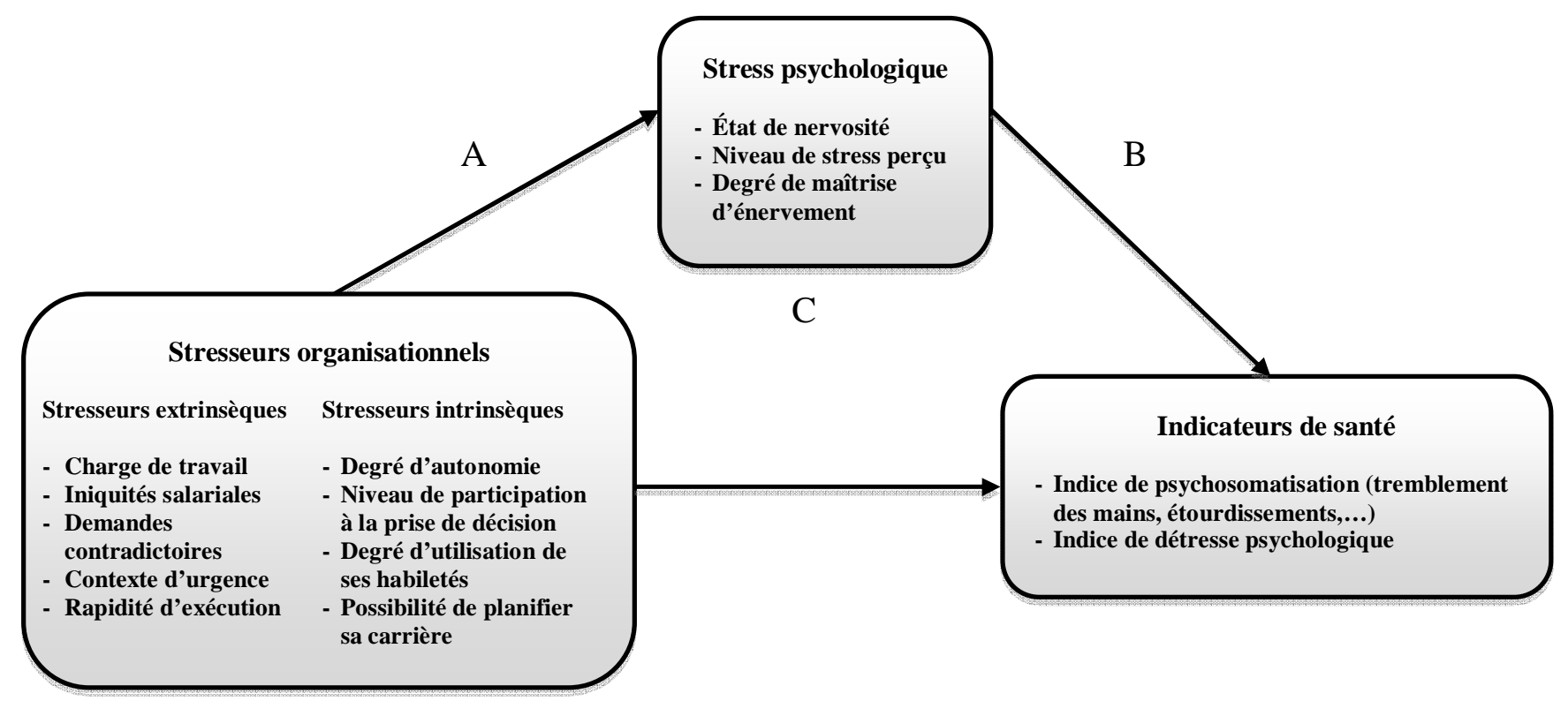

\subsection{L'effet médiateur du stress psychologique}

Les figures 3 et 4 présentent les relations entre les stresseurs organisationnels, le stress psychologique et les indicateurs de santé observées dans notre étude. Il est à noter que les résultats tiennent compte de certaines caractéristiques des travailleurs comme le genre, l'âge, le statut civil et le degré de scolarité de sorte que les relations entre les stresseurs, le stress et les indicateurs de santé ne découlent pas des différences entre les individus pour ces caractéristiques personnelles. De plus, les effets des stresseurs organisationnels ainsi que du stress psychologique sur les indicateurs de santé sont similaires pour les symptômes psychosomatiques et la détresse psychologique et ne seront donc pas présentés de façon séparée. Par ailleurs, seules les relations significatives sur un plan statistique sont illustrées dans ces figures.

Figure 3 : Les effets des stresseurs extrinsèques

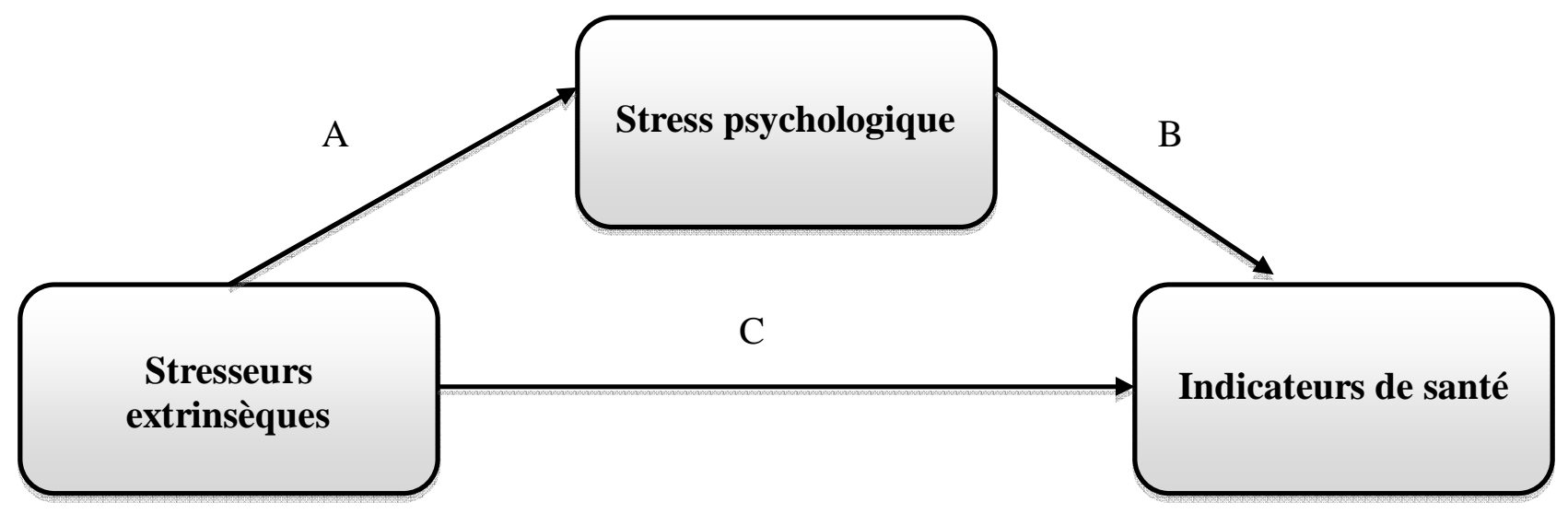


Les travailleurs se sentent donc plus stressés tant par les conditions plus objectives du travail (exigences élevées et contexte d'exécution) que par la faible possibilité d'utiliser leurs habiletés et de faire preuve d'autonomie pour rencontrer ces exigences.

La relation (A) dans les deux figures démontre que tant les stresseurs intrinsèques que les stresseurs extrinsèques affectent le niveau de stress psychologique ressenti par les travailleurs. Les travailleurs se sentent donc plus stressés tant par les conditions plus objectives du travail (exigences élevées et contexte d'exécution) que par la faible possibilité d'utiliser leurs habiletés et de faire preuve d'autonomie pour rencontrer ces exigences. La relation (B) illustre qu'en contrepartie, le stress psychologique affecte significativement les deux indicateurs de santé utilisés. Ceci signifie que les travailleurs qui sont plus stressés présentent plus de symptômes psychosomatiques ainsi qu'une détresse psychologique plus élevée.

Les résultats démontrent toutefois une différence importante dans l'action des stresseurs organisationnels sur les indicateurs de santé. Ainsi, les stresseurs extrinsèques de nature plus objective (figure 3) ont une action directe sur les indicateurs de santé qui s'ajoute à l'effet indirect ou médiateur du stress psychologique. En présence de stresseurs extrinsèques élevés, les travailleurs auront un moins bon état de santé lorsque leur niveau de stress augmente. De plus, leur état de santé sera également affecté même si leur niveau de stress n'est pas plus élevé. Pour leur part, les stresseurs intrinsèques (figure 4) affectent l'état de santé uniquement par l'intermédiaire du stress psychologique et ne présentent pas les effets directs observés pour les stresseurs extrinsèques. Les stresseurs intrinsèques affectent ainsi l'état de santé uniquement chez les travailleurs qui ressentent un niveau plus élevé de stress. En d'autres termes, le fait de ne pas utiliser ses habiletés ou de ne pas pouvoir faire preuve d'autonomie dans son travail n'aura des répercussions sur l'état de santé que dans la mesure où les travailleurs ressentent un niveau élevé de stress.

Le fait de ne pas utiliser ses habiletés ou de ne pas pouvoir faire preuve d'autonomie dans son travail n'aura des répercussions sur l'état de santé que dans la mesure où les travailleurs ressentent un niveau élevé de stress.

Figure 4 : Les effets des stresseurs intrinsèques

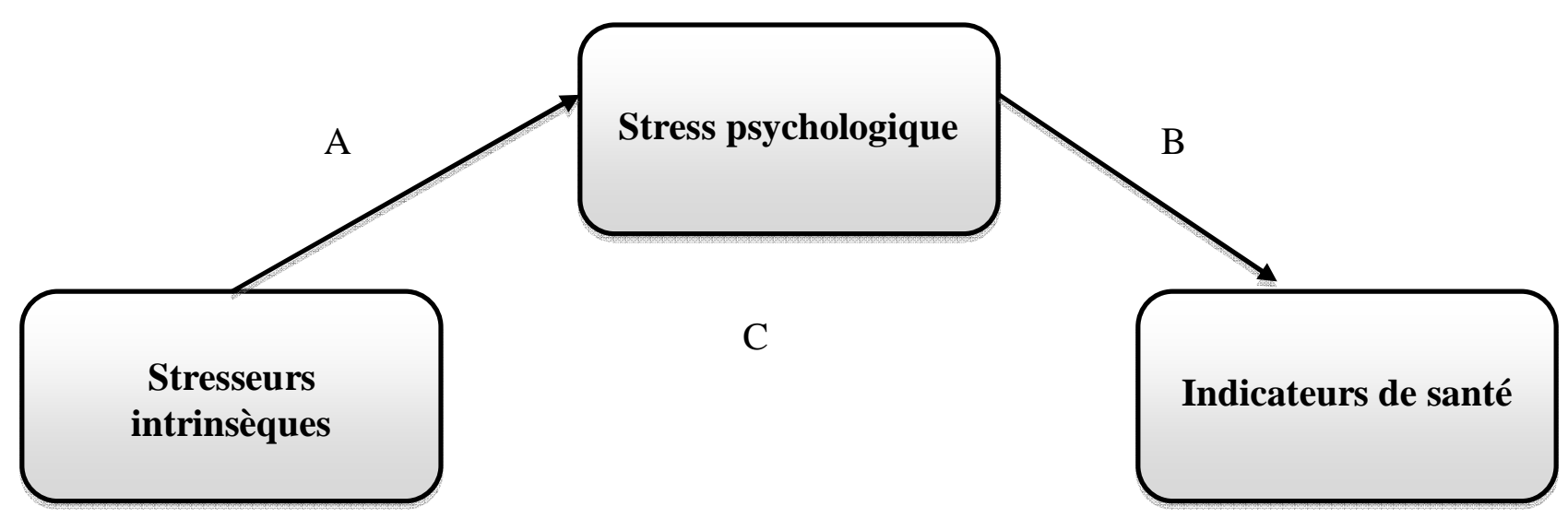




\section{CONCLUSION}

\section{La présente démarche démontre qu'une partie de l'effet des stresseurs} organisationnels passe par l'intermédiaire du stress psychologique. Autrement dit, le stress psychologique agit comme médiateur alors que les stresseurs organisationnels agissent sur le stress psychologique, lequel affecte à son tour significativement l'état de santé : c'est l'effet domino.

Cette étude démontre de façon originale et plus précise que les stresseurs organisationnels affectent la santé physique et mentale des travailleurs. Tout en étant conforme à la plupart des travaux de recherche qui se concentrent sur l'association directe entre ces stresseurs et la santé des travailleurs, cette étude va au-delà en ayant réalisé une autopsie de cette association. En effet, la présente démarche démontre qu'une partie de l'effet des stresseurs organisationnels passe par l'intermédiaire du stress psychologique. Autrement dit, le stress psychologique agit comme médiateur alors que les stresseurs organisationnels agissent sur le stress psychologique, lequel affecte à son tour significativement l'état de santé : c'est l'effet domino.

La perception individuelle via le stress psychologique est centrale dans l'influence des stresseurs intrinsèques sur l'état de santé alors qu'elle est plus accessoire dans l'influence des stresseurs extrinsèques.

Cet effet médiateur du stress psychologique est plus probant dans le cas des stresseurs intrinsèques alors que la médiation est parfaite tandis qu'elle est partielle dans le cas des stresseurs extrinsèques. Nous constatons ainsi un effet différencié de l'évaluation cognitive eu égard à la nature des stresseurs. La perception individuelle via le stress psychologique est centrale dans l'influence des stresseurs intrinsèques sur l'état de santé alors qu'elle est plus accessoire dans l'influence des stresseurs extrinsèques. Ainsi, les conditions objectives posées par les exigences du travail et le contexte dans lequel il s'exerce (p. ex. une charge de travail élevée ou un contexte d'urgence) affectent la santé des travailleurs par-delà la perception des caractéristiques nocives des conditions de travail. En fait, l'effet délétère des stresseurs extrinsèques au travail se ferait en deçà de la perception, du moins partiellement et aurait une incidence directe sur la santé peu importe les caractéristiques personnelles du travailleur.

\section{L'effet délétère des stresseurs extrinsèques au travail se ferait en deçà de la perception, du moins partiellement et aurait une \\ incidence directe sur la santé peu importe les caractéristiques personnelles du travailleur.}

Il est important de comprendre le processus subjectif par lequel les travailleurs identifient les stresseurs de leur milieu de travail et pourquoi certains semblent plus vulnérables que d'autres. Ceci pose la question de l'efficacité des interventions sur la problématique de la santé et du stress au travail. Les mesures centrées sur les individus pour les amener à mieux gérer leur niveau de stress ou atténuer les effets sur leur santé ne sauraient être suffisantes. Particulièrement, nos résultats expliquent l'inefficacité relative des stratégies d'adaptation (surtout cognitives) lorsque les travailleurs ont à transiger avec des stresseurs organisationnels de nature extrinsèque. Les interventions doivent cibler tant les conditions de travail qui agissent directement sur la santé pour les ramener à un niveau plus acceptable que de donner la possibilité aux individus de disposer de la marge de manœuvre nécessaire pour faire face aux exigences de leur travail. 


\section{Bibliographie}

${ }^{1}$ Laplante, N., Gosselin, E. et Lauzier, M. (2008). «Dynamique transactionnelle du stress et santé des travailleurs : examen du rôle médiateur du stress psychologique », Actes du 15e Congrès de l'Association Internationale de Psychologie du Travail de Langue Française (AIPTLF), Québec. Presses de l'Université du Québec. pp. 1-12. (CD-ROM).

${ }^{2}$ Brun, J.-P., Biron, C., Martel, J. et Ivers, H. (2003). Évaluation de la santé mentale au travail : une analyse des pratiques de gestion des ressources humaines. Rapport R-342, Montréal, Québec : IRSST. 100p.

${ }^{3}$ Karasek, R. et Theorell, T. (1990). Healthy Work: Stress, Productivity and the Reconstruction of Working Life. New York, NY : Basic Books. 400p.

${ }^{4}$ Siegrist, J. (1996). « Adverse Health Effects of HighEffort/Low-Reward Conditions », Journal of Occupational Health Psychology, 1 (1), pp. 27-41.

${ }^{5}$ Lazarus, R. S. (1999). Stress and Emotions: a New Synthesis. New York, NY : Springer. 340p.

${ }^{6}$ Gosselin, E., Lemyre, L., Corneil, W., Barelle, J. et Beauregard, N. (2007). «Lien entre les stresseurs organisationnels et l'état de santé : vérification du rôle médiateur du stress psychologique », Actes $d u$ $50^{e}$ Congrès de l'Association des Sciences Administratives du Canada (ASAC), Ottawa, Ontario, Canada. pp. 112-128 


\title{
Les études de cycles supérieurs à l'UQAC
}

ARTS ET LETTRES - Programme court en didactique du français langue maternelle • Maîtrise en art• Diplôme en didactique du français langue maternelle $\bullet$ Maîtrise en études littéraires • Maîtrise en linguistique $\bullet$ Doctorat en lettres

SCIENCES HUMAINES • Programme court en études régionales • Diplôme en sciences infimières - Maîtrise en médecine expérimentale génétique des populations • Maîtrise en études et interventions régionales • Doctorat en développement régional • Maîtrise en sciences infirmières • Maîtrise en travail social $\bullet$ Programme court en éthique $\bullet$ Programme court en études religieuses $•$ Diplôme en éthique de société - Maîtrise en théologie pratique • Doctorat en théologie pratique - Microprogramme en théologie pratique $\bullet$ Programme court d'accompagnement des aînés

SCIENCES DE L'ÉDUCATION ET DE PSYCHOLOGIE • Diplôme en intervention éducative • Diplôme en enseignement collégial $\bullet$ Maîtrise en éducation • Doctorat en psychologie profil intervention • Doctorat en éducation • Programme court en intervention éducative $\bullet$ Programme court en administration scolaire • Diplôme en administration scolaire

SCIENCES ÉCONOMIQUES ET ADMINISTRATIVES - Programme court en gestion de projet - Diplôme en gestion de projet - Maîtrise en gestion de projet • Maîtrise en gestion des organisations - Maîtrise en administration des affaires pour cadres • Diplôme en sciences comptables

SCIENCES APPLIQUÉES • Maîtrise en ingénierie • Doctorat en ingénierie • Diplôme en sciences de la Terre $\bullet$ Maîtrise en sciences de la Terre $\bullet$ Doctorat en ressources minérales

SCIENCES FONDAMENTALES - Maîtrise en ressources renouvelables - Doctorat en sciences de l'environnement • Diplôme en éco-conseil

INFORMATIQUE ET MATHÉMATIQUES

- Diplôme en informatique appliquée • Maîtrise en informatique

\section{2,5 millions \$}

pour les étudiantes et les étudiants

en support et formation à la recherche

\section{Consultez nos programmes}

\author{
www uqac.ca
}

\section{UQAC}

UNIVERSITÉ DU QUÉBEC À CHICOUTIMI

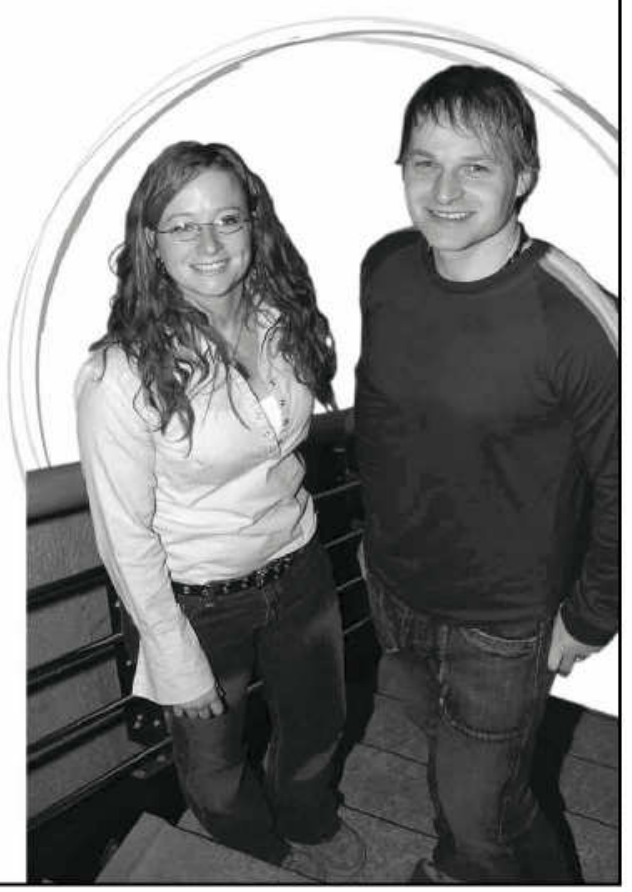

